\title{
Phosphoprotein associated with glycosphingolipid microdomains 1 inhibits the proliferation and invasion of human prostate cancer cells in vitro through suppression of Ras activation
}

\author{
WENJUAN YU ${ }^{1,2}$, YUEWEI WANG $^{3}$, MIAOZI GONG ${ }^{2}$, FEI PEI $^{2}$ and JIE ZHENG ${ }^{2}$ \\ ${ }^{1}$ Department of Pathology, The Affiliated Hospital of Medical College, Qingdao University, Qingdao; \\ ${ }^{2}$ Department of Pathology, Health Science Center, Peking University, Beijing; ${ }^{3}$ Department of Vascular Surgery, \\ The Affiliated Hospital of Medical College, Qingdao University, Qingdao, P.R. China
}

Received February 14, 2012; Accepted March 26, 2012

DOI: $10.3892 /$ or.2012.1848

\begin{abstract}
Phosphoprotein associated with glycosphingolipid microdomains 1 (PAG) is an important negative regulator of immune signaling in T lymphocytes. However, newly emerging evidence has indicated that PAG may play important roles in tumor cells. Our previously reported cDNA microarray experiments identified PAG as a gene down-regulated in the high metastatic potential prostate cancer cell line PC-3M-1E8. In this study, we investigated the role of PAG in the proliferation, invasion and metastasis of prostate cancer cells and the underlying mechanisms. We confirmed that the expression of PAG at both the mRNA and protein levels was low in PC-3M-1E8 and DU145 cells compared to low metastatic potential prostate cancer cells PC-3M-2B4. In addition, we demonstrated that the reintroduction of PAG to PC-3M-1E8 and DU145 cells led to reduced proliferation through cell cycle arrest, decreased anchorage-independent growth and reduced invasion ability of tumor cells in vitro. This is the first report demonstrating that PAG inhibits the proliferation and invasion potential of prostate cancer cells via the interaction with RasGAP to recruit RasGAP to the cell membrane, where RasGAP hydrolyzes GTP to GDP, reduces the level of activated Ras, and ultimately suppresses the activation of ERK1/2, cyclin D1 and other effectors of the Ras signaling pathway. Morphologically, we observed that PAG could diminish the formation of pseudopodia on the cell surface and redistribute the intracellular F-actin in PC-3M-1E8 cells, which directly leads to the decreased invasion and metastasis potential of tumor cells. Taken together, these results suggest that PAG acts to inhibit the development and metastasis of prostate cancers and represents a novel therapeutic target for prostate cancer.
\end{abstract}

Correspondence to: Dr Jie Zheng, Health Science Center, Peking University, No. 38, Xueyuan Road, Haidian District, Beijing 100191, P.R. China

E-mail: zhengjie@bjmu.edu.cn

Key words: prostate cancer, cancer metastasis, gene, PAG, RasGAP

\section{Introduction}

Prostate cancer frequently develops in older males. It is the second leading cause of cancer death in American men. Prostate cancer has serious impact on the survival and quality of living for patients due to its high potential of metastasis to other parts of the body. Therefore, deeper understanding of the mechanisms responsible for the metastasis of prostate cancer is important for the prevention and therapy of prostate cancer. To identify important genes that contribute to metastasis of prostate cancer, previously we performed cDNA microarrays to screen differentially expressed genes between two PC-3M prostate cell lines, i.e., PC-3M-1E8 (a high metastatic subline) and PC-3M-2B4 (a low metastatic subline) (1). Phosphoprotein associated with glycosphingolipid microdomains 1 (PAG) was one of the down-regulated genes in the high metastatic PC-3M-1E8 subline.

PAG, also called Csk binding protein (Cbp), is a ubiquitously expressed transmembrane adaptor protein. The structure of PAG is characterized by a substantial intracytoplasmic region that contains multiple tyrosine residues which can be phosphorylated by Src-family kinases (2). In vitro, PAG could bind a number of signaling molecules via their SH2 domains, such as Lyn, Fyn and Csk, a negative regulator of Src-family kinases (3). Previous studies showed that PAG is an important negative regulator of immunoreceptor signaling in $\mathrm{T}$ lymphocyte $(4,5)$. The binding of PAG and Csk induced the recruitment of Csk to the membrane and suppressed the activity of membrane associated Src-family kinases, therefore playing an important role in the regulation of essential cellular activity to keep the resting state of T lymphocytes $(6,7)$. In addition, PAG was able to inhibit the malignancy transformation and proliferation of tumor cells by inhibiting the activation of Src (8). In the stimulated T lymphocyte PAG could interact with RasGAP (Ras GTP activation protein) as revealed by immunoprecipitation, indicating that PAG may negatively regulate Ras activity (9).

The recruitment of RasGAP to the membrane stimulates the rapid hydrolysis of Ras-GTP formed in the lipid rafts, thus effectively blocking Ras activation. RasGAP could perform this regulatory function because it possesses two SH2 domains and could bind PAG via its N-terminal SH2 domain $(10,11)$. 
Therefore, we speculate that the interaction of RasGAP with PAG is not exclusive in T lymphocytes, but also in other kinds of cells including tumor cells, which could lead to the inhibition of Ras activity and the alteration of biological behaviors of tumor cells. However, this hypothesis has never been studied and reported so far.

The present study aimed to investigate the role of PAG in the proliferation, invasion and metastasis of prostate cancer cells and the underlying mechanisms. Our results demonstrated that increased expression of PAG could inhibit the proliferation and invasion of PC-3M-1E8 and DU145 cells in vitro through interacting with RasGAP and regulating the effectors of Ras signaling, such as Ras, p-ERK and cyclin D1.

\section{Materials and methods}

Cell culture. PC-3M-1E8, a variant subline of PC-3M with high metastatic potential, and PC-3M-2B4, a variant subline of PC-3M with low metastatic potential, and DU145, a high metastatic prostate cancer cell line, were cultured in RPMI-1640 medium (Gibco) supplemented with $10 \%$ heat-inactivated fetal bovine serum (Gibco) at $37^{\circ} \mathrm{C}$ in a humidified incubator with $5 \% \mathrm{CO}_{2}$.

Real-time $R T-P C R$. Total RNA was extracted from the PC-3M-1E8, PC-3M-2B4 and DU145 cells by TRIzol reagent (Invitrogen), and used as the template for the synthesis of cDNA by M-MLV reverse transcriptase (Promega). The mRNA level of PAG was detected by ABI PRISM 7500 fluorescent quantitative polymerase chain reaction instrument. The forward primer (GATGTTCAGCCGTTCAGTTAC) and reverse primer (TCTGGACTTCCTCGTAATGC) were designed using primer premier 5.0 software. The reaction conditions were: $95^{\circ} \mathrm{C}$ for $5 \mathrm{~min}$; followed by 40 cycles at $95^{\circ} \mathrm{C}$ for $1 \mathrm{~min}, 60^{\circ} \mathrm{C}$ for $30 \mathrm{sec}$ and $72^{\circ} \mathrm{C}$ for $30 \mathrm{sec}$.

Western blot analysis. PC-3M-1E8, PC-3M-2B4 and DU145 cells were collected and resuspended in 2X SDS cell lysis solution (100 mM Tris-HCl pH 6.8, 4\% SDS, 20\% glycerol, $200 \mathrm{mM}$ DTT, $0.2 \%$ bromophenol blue). The cell lysate was separated by sodium dodecyl sulfate-polyacrylamide gel electrophoresis (SDS-PAGE) and then transferred to nitrocellulose membranes. The membranes were subsequently probed by PAG antibody (1:400, Abcam) and tubulin antibody (1:400, Neomarker), and secondary antibody. The membranes were developed using ECL chemiluminescence reagent (Pierce).

Plasmids and transfection. The cDNA of human PAG was amplified from total RNA isolated from PC-3M-2B4 cells by RT-PCR (forward primer: CGGAATTCGCCACCATGGGGC CCG CGGGGAGCCT, reverse primer: CCGCTCGAGCTA GAGCCTGGTAATATCTCTG CCTTGCTGCAAGTCAC). The PCR products were cloned into the eukaryotic expression vector pcDNA3 (Invitrogen) by EcoRI and XhoI sites. The recombined plasmid pcDNA3-PAG was verified by sequencing and prepared by Pureyield ${ }^{\mathrm{TM}}$ plasmid Midprep kit (Promega). PC-3M-1E8 and DU145 cells were transfected with pcDNA3PAG using Lipofectamine ${ }^{\mathrm{TM}} 2000$ (Invitrogen) and selected with G418 (600 $\mu \mathrm{g} / \mathrm{ml}$, Biochem). Four clones of each cell with high PAG expression level identified by western blot were mixed for the subsequent studies. PC-3M-1E8 and DU145 transfected with pcDNA3 and parental PC-3M-1E8 and DU145 cells were used as the controls.

Cell proliferation assay. MTT assay was performed to evaluate cell proliferation as previously described (12). Briefly, cells were seeded in a 96-well-plate at $1.0 \times 10^{3}$ cells/well in triplicate. MTT solution $(20 \mu \mathrm{l})(5 \mathrm{mg} / \mathrm{ml}$, Sigma) was added into each well and $150 \mu \mathrm{l}$ DMSO was added $4 \mathrm{~h}$ later. Then the plate was measured for the absorbance at $570 \mathrm{~nm}$ by ELISA.

Soft agar colony formation assay. Low melting-point agarose (1.2\%) and 2X DMEM culture solution with $20 \%$ fetal bovine serum were mixed to be used as the bottom-layer agar in the 60-mm culture dishes. Single-cell suspensions of $4 \times 10^{4}$ cells were subcultured into $3 \mathrm{ml}$ DMEM containing $20 \%$ fetal bovine serum and $0.7 \%$ low melting-point agarose. Twenty days after plating, the number of colonies containing $>50$ cells was counted under inverted microscope.

Flow cytometry for cell cycle analysis. Cells were digested by $10 \%$ trypsin and washed twice in cold PBS and subsequently fixed by $75 \%$ alcohol at $4^{\circ} \mathrm{C}$ for $24 \mathrm{~h}$. Three hundred-mesh nylon net was applied to filter the cells and then $10 \mu$ l RNase was added and incubated at $37^{\circ} \mathrm{C}$ for $30 \mathrm{~min}$. Following staining with PI, samples were subjected to flow cytometry analysis (BD FACSCalibur).

Flow cytometry for analysis of apoptosis. The Apoptosis Assay kit (Gene Research Center of Peking University) was used to evaluate prostate cancer cell apoptosis. Cells were digested by $10 \%$ trypsin and washed twice in cold PBS. Three hundred-mesh nylon net was used to filter the cells. Cells were resuspended in $200 \mu \mathrm{l}$ binding buffer. Then $10 \mu \mathrm{l}$ Annexin-V-FITC was added and incubated at $4^{\circ} \mathrm{C}$ for $30 \mathrm{~min}$. Cells were stained with $5 \mu \mathrm{l}$ propidium iodide (PI) and subjected to flow cytometry analysis.

Invasion assay. The conditional medium of NIH3T3 cells was used as the chemotactic factor in the inferior part of the Boyden chambers. A polycarbonate $8-\mu \mathrm{m}$ thick millipore membrane was placed between the superior and inferior chambers. Matrigel $(50 \mu \mathrm{l})(1 \mathrm{~g} / \mathrm{l}, \mathrm{BD})$ was evenly distributed on the membrane. After complete polymerization of the matrigel, $\sim 2 \times 10^{5}$ cells were seeded into the superior chamber of the well. Then the cells were cultivated at $37^{\circ} \mathrm{C}$ in $5 \% \mathrm{CO}_{2}$ for $8 \mathrm{~h}$. The cells that crossed the membrane were fixed by methanol and stained by hematoxylin and eosin (H\&E), and counted under a light microscope.

Immunoprecipitation. For immunoprecipitation, $2 \times 10^{6}$ cells were lysed in $500 \mu \mathrm{l}$ cold NP-40 lysis buffer (1\% NP-40, $50 \mathrm{mM}$ Tris-buffer saline $\mathrm{pH} 8.2,100 \mathrm{mM} \mathrm{NaCl}, 5 \mathrm{mM}$ EDTA) containing $5 \mu \mathrm{l}$ protease inhibitor cocktail and $5 \mu \mathrm{l}$ phosphatase inhibitor for $30 \mathrm{~min}$ and then centrifuged for $15 \mathrm{~min}$ at $4^{\circ} \mathrm{C}, 12000 \mathrm{rpm}$ to collect the supernatant. PAG antibody (2 $\mu \mathrm{g}$, Abcam) and protein A agarose beads (Santa Cruz) were added into the supernatant and the mixture was rotated at $4^{\circ} \mathrm{C}, 100 \mathrm{rpm}$ for $12 \mathrm{~h}$. In the control group, PAG antibody was replaced by mouse IgG. Beads were washed three times with PBS containing protease inhibitor cocktail and phosphatase inhibitor, resuspended in 2X SDS loading 
A

Expression of PAG at mRNA level

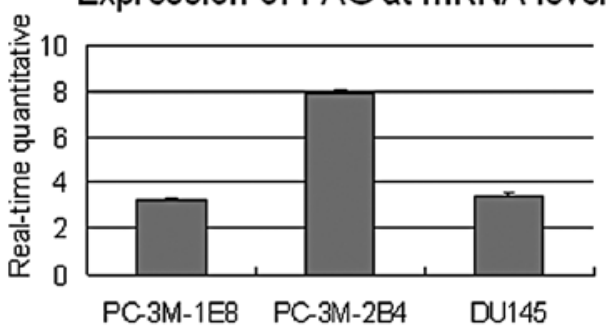

B

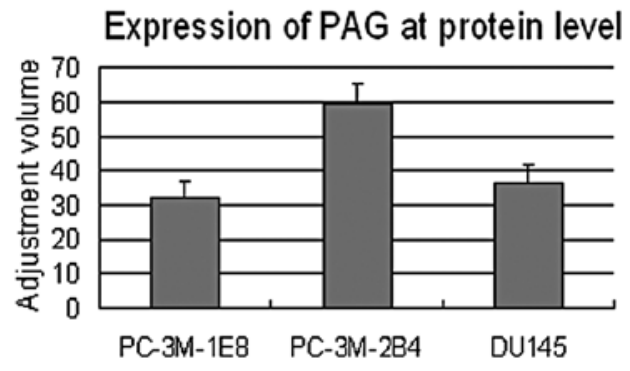

\section{C}

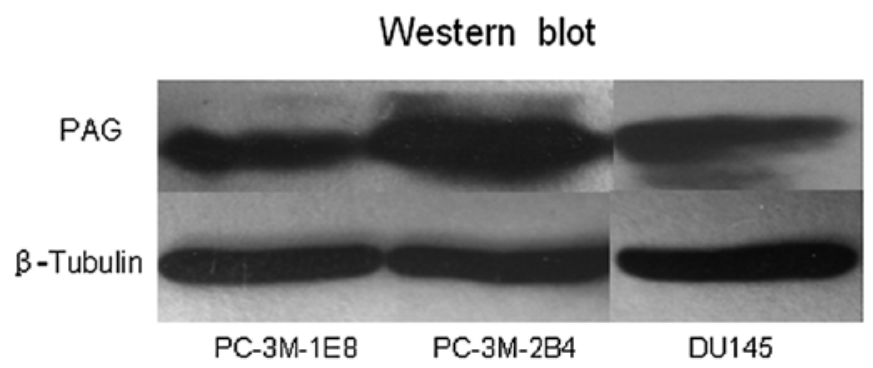

Figure 1. (A) The results of real-time quantitative RT-PCR. (B and C) The results of western blot. (A and B) mRNA and protein expression levels of PAG in PC-3M-1E8 and DU145 cells were lower than those in PC-3M-2B4 cells. PAG migrated as a thick band with a molecular weight $\sim 60-70 \mathrm{kDa}$.

buffer (100 mM Tris-HCl pH 6.8, 4\% SDS, $20 \%$ glycerol, 200 mM DTT, $0.2 \%$ bromophenol blue) and boiled for $10 \mathrm{~min}$. After centrifuging, the supernatant was collected for western blot analysis using RasGAP antibody (1:300, Abcam).

GST pull-down assay. The expression of the fusion protein pGEX-GST-Raf1-Ras-binding-domain (RBD) (amino acids 51-131 of human Raf-1) in JM109 E. coli was induced by IPTG (final $1 \mathrm{mmol} / \mathrm{l}$, Gold Biotechnology). SDS-PAGE and coomassie brilliant blue staining were performed using $20 \mu 1$ lysate of the bacterium to estimate the expression of the fusion protein. Total bacterial lysate was mixed with 30 $\mu \mathrm{l}$ GST-sepharose 4 beads (Pharmacia) and incubated for $1 \mathrm{~h}$ at room temperature. After being washed in PBS three times, the beads were mixed with $300 \mu \mathrm{l}$ cell lysate $\left(\sim 2 \times 10^{6}\right.$ cells $)$ and incubated at $4^{\circ} \mathrm{C}$ for $12 \mathrm{~h}$. Then the beads were washed three times with NP-40 solution, resuspended in 2X SDS loading buffer and boiled for $10 \mathrm{~min}$. After centrifuging, the supernatant was collected for western blot analysis using pan-Ras antibody (1:500, Cell Signaling).

Western blot analysis. PC-3M-1E8 cells were lysed in cold NP-40 lysis buffer and the cell lysate was collected for western blot analysis. The antibodies used were as follows: anti-ERK1/2 (1:1000), anti-p-ERK1/2 (1:200), anti-cyclin D1 (1:500), antiP21 ${ }^{\text {WAFI/CIP1 }}$ (1:300) and anti-p-AKT (1:500). All of them were purchased from Santa Cruz Biotechnology.

TRITC-phalloidine staining. The cell suspension was dropped onto the sterile glass slide and incubated at $37^{\circ} \mathrm{C}$ for $24 \mathrm{~h}$ in a humidified incubator with $5 \% \mathrm{CO}_{2}$. Then the slides were fixed in cold acetone for $30 \mathrm{~min}$. After being washed in PBS three times, the slides were treated with $0.2 \%$ Triton X-100 for $2 \mathrm{~min}$ for adequate permeabilization. Next the slides were washed by PBS three times and stained by phalloidine labeled by tetramethylrhodamine-5-(and-6)-isothiocyanate (TRITC) (1:50 dilution, Sigma) for $45 \mathrm{~min}$ at room temperature in the dark. Finally, the slides were mounted by the mixture of glycerin and PBS at equal volume. The cell morphology and F-actin arrangement were observed under a laser passing confocal microscope. F-actin was stained red.

Statistical analysis. Statistical analysis was performed using SPSS13.0 software. $\mathrm{P}<0.05$ was considered statistically significant.

\section{Results}

Low expression of PAG in high metastatic prostate cancer cells. To confirm our previous cDNA microarray results showing that PAG was down-regulated in the PC-3M-1E8 cells compared to PC-3M-2B4 cells, we performed real-time quantitative RT-PCR and western blot analysis to evaluate the expression level of PAG in these prostate cancer cells. Realtime RT-PCR analysis showed that the expression of PAG at mRNA level was twice that in PC-3M-2B4 cells than in PC-3M-1E8 and DU145 (P<0.5, Fig. 1A). Western blot analysis further demonstrated that the expression of PAG at protein level was low in PC-3M-1E8 and DU145 cells compared to that in $\mathrm{PC}-3 \mathrm{M}-2 \mathrm{~B} 4(\mathrm{P}<0.5$, Fig. 1B and $\mathrm{C})$. Taken together, these results demonstrate that PAG is down-regulated in high metastatic prostate cancer cells, suggesting that PAG may inhibit prostate cancer development and metastasis.

PAG inhibits the proliferation and invasion of high metastatic prostate cancer cells. To provide experimental evidence that PAG inhibits prostate cancer development and metastasis, first we performed MTT assay and soft-agar colony-formation assay to examine the proliferation ability of PC-3M-1E8 and DU145 cells that exogenously expressed 
A

MTT assay of PC-3M-1E8

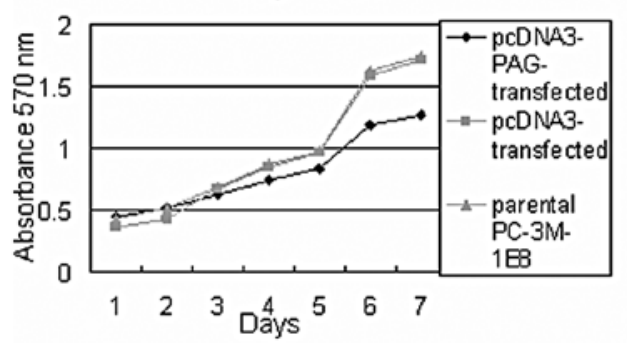

B

Colony-formation assay of PC-3M-1E8

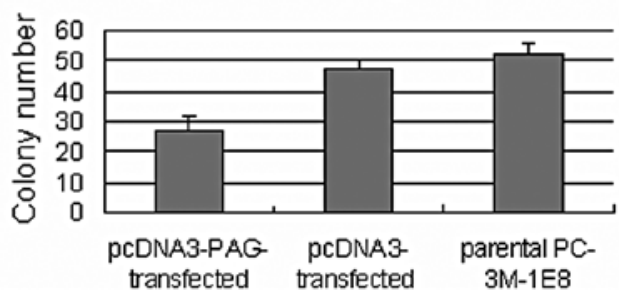

MTT assay of DU145

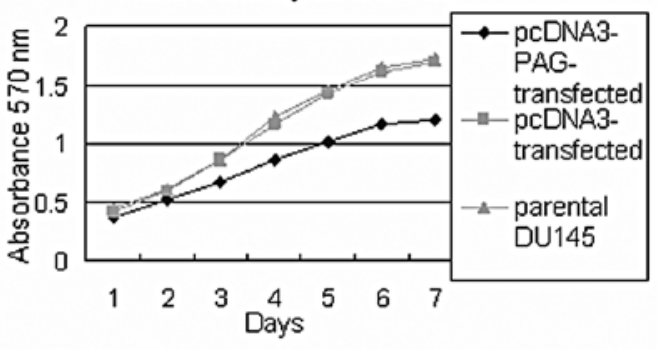

Colony-formation assay of DU145

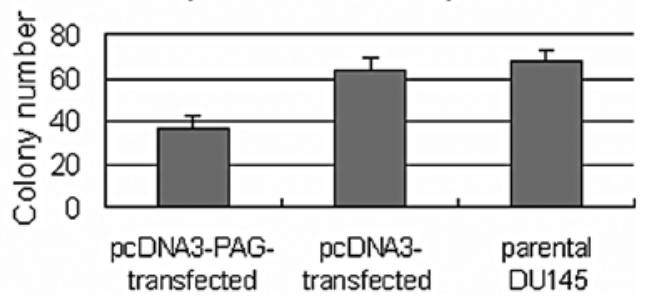

Figure 2. The result of MTT assay (A) and soft-agar colony-formation assay (B). PC-3M-1E8 and DU145 cells expressing exogenous PAG grew more slowly and formed fewer colonies than the control cells under the same conditions.
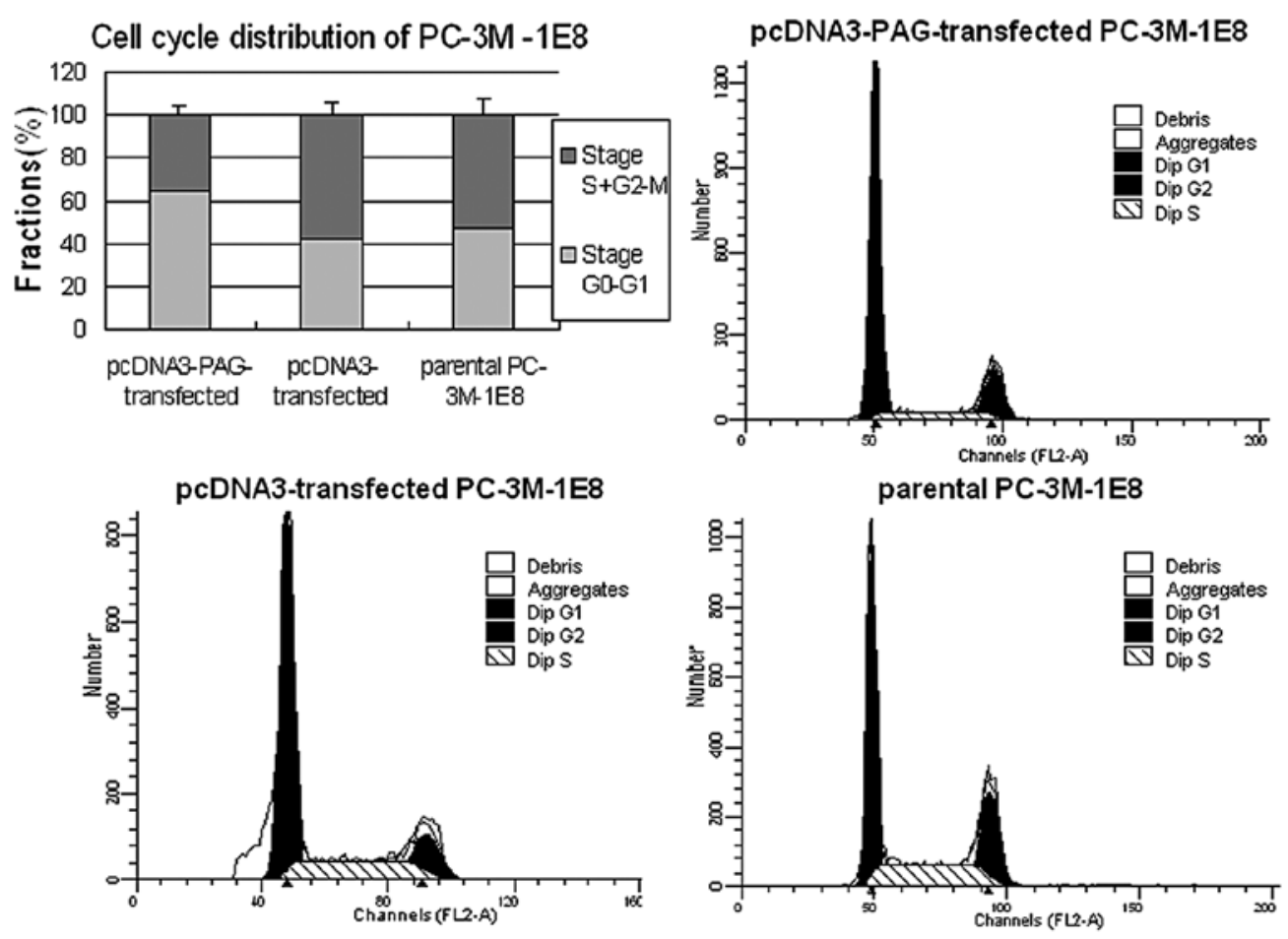

Figure 3. The distribution of the cell cycle in different groups of PC-3M-1E8 cells. Higher percentage of cells was distributed in stage $\mathrm{G}_{0}-\mathrm{G}_{1}$ in $\mathrm{PC}-3 \mathrm{M}-1 \mathrm{E} 8$ expressing exogenous PAG than in the controls.

PAG. The results showed that PC-3M-1E8 and DU145 cells expressing exogenous PAG grew more slowly and formed fewer colonies than the control cells under the same circumstance $(\mathrm{P}<0.5$, Fig. 2). Next we performed flow cytometry analysis to investigate the cell cycle progression and apoptosis of these cells. The results demonstrated that higher percentage of cells was distributed in stage $G_{0}-G_{1}$ in PC-3M-1E8 (Fig. 3) and DU145 cells (Fig. 4) expressed exogenous $\mathrm{PAG}$ more than in the controls $(\mathrm{P}<0.5)$. However, no significant difference in the apoptosis rate was found between PC-3M-1E8 and DU145 cells expressing exogenous PAG and the controls ( $\mathrm{P}>0.5$, Fig. 5). Taken together, these results indicate that the decreased proliferation of PC-3M-1E8 and DU145 cells upon exogenous expression 

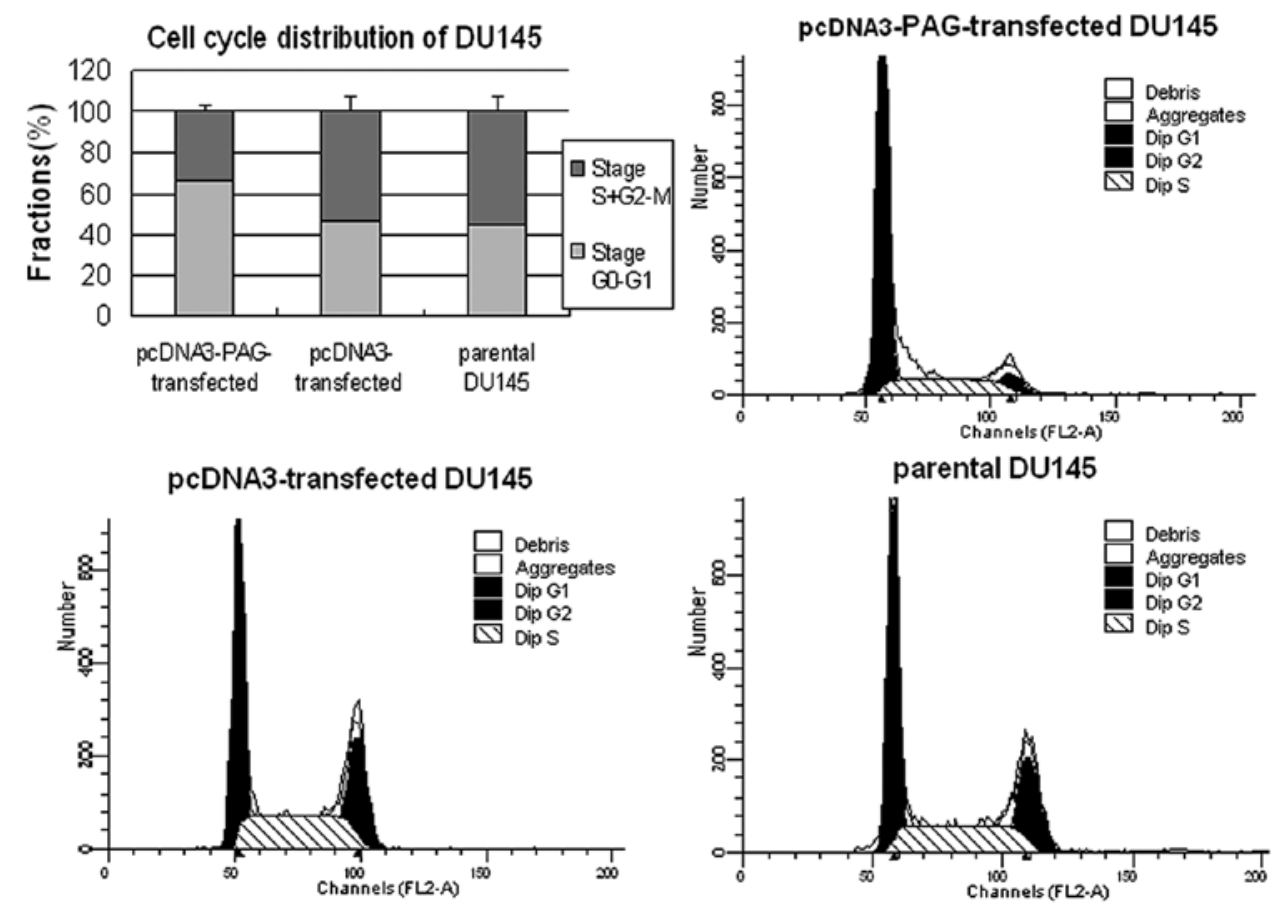

Figure 4. The distribution of the cell cycle in different groups of DU145 cells. The cell numbers in stage $\mathrm{G}_{0}-\mathrm{G}_{1}$ increased in PAG-transfected DU145 cells compared with the controls.
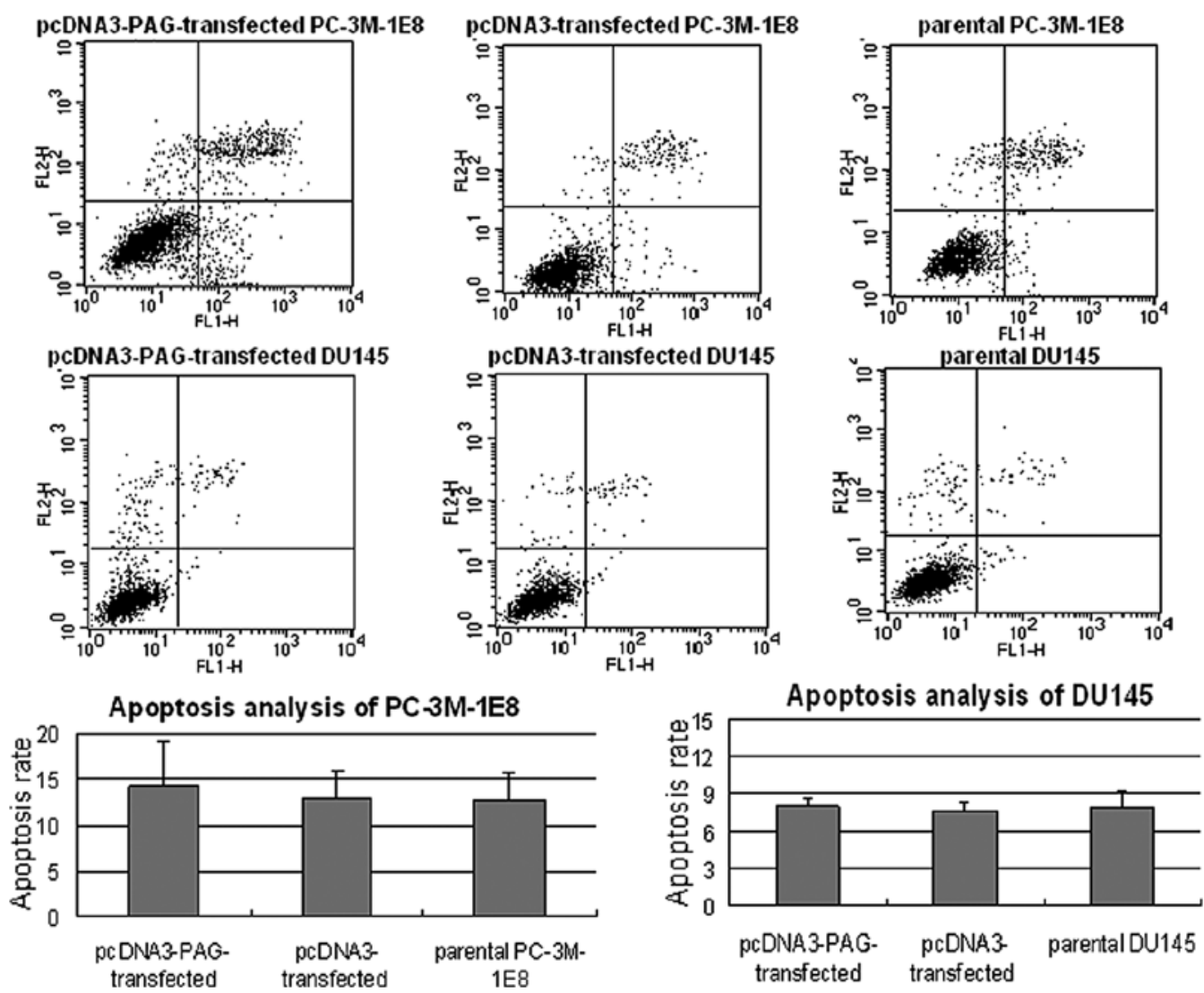

Figure 5. The apoptosis rate of different groups of PC-3M-1E8 and DU145 cells. No significant difference in the apoptosis rate was found between PC-3M-1E8 and DU145 cells expressing exogenous PAG and the controls.

of PAG is attributed to the arrest of cell cycle rather than the promotion of apoptosis.
Furthermore, we employed matrigel invasion assay to examine the invasion ability of PC-3M-1E8 and DU145 cells 

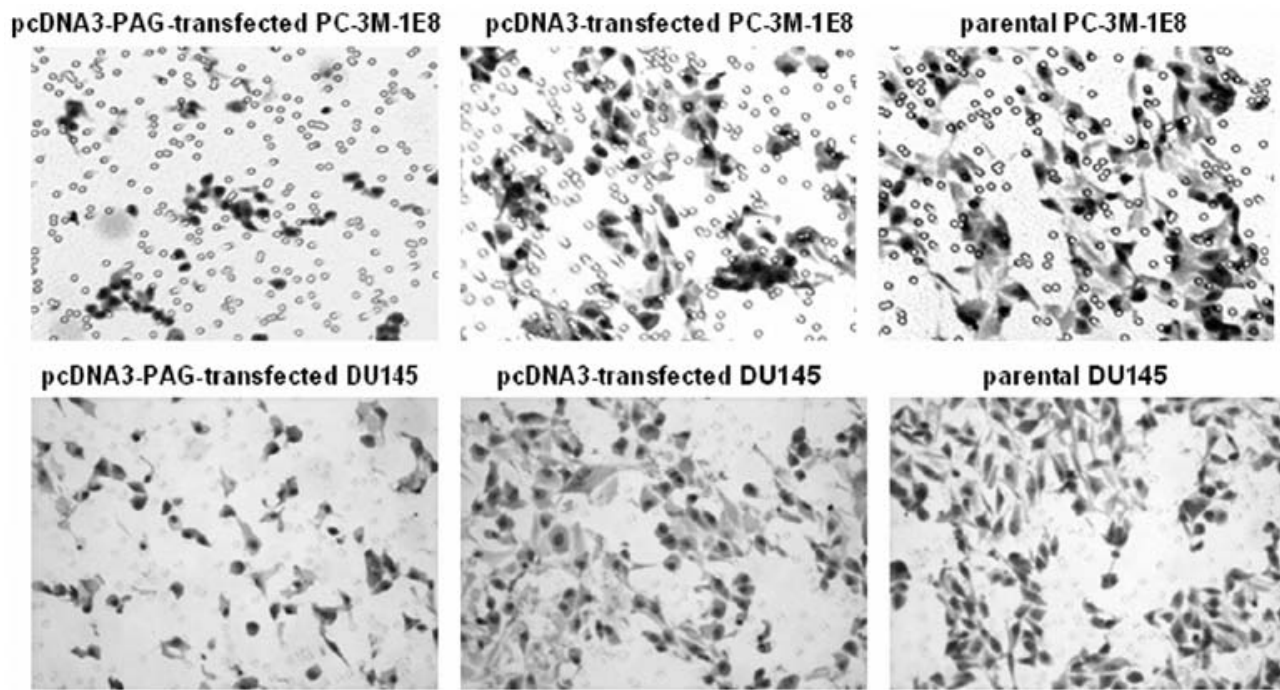

pcDNA3-transfected DU145

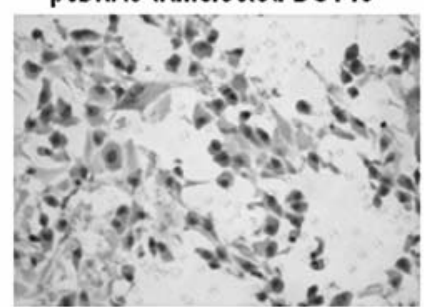

parental DU145

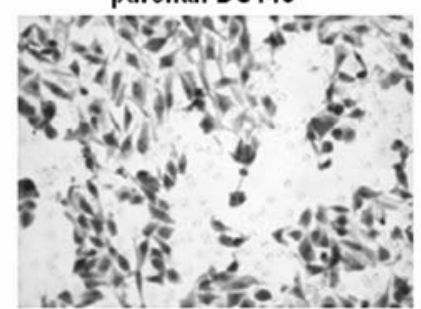

Invasion as say of PC $3 \mathrm{M}-1 \mathrm{E} 8$

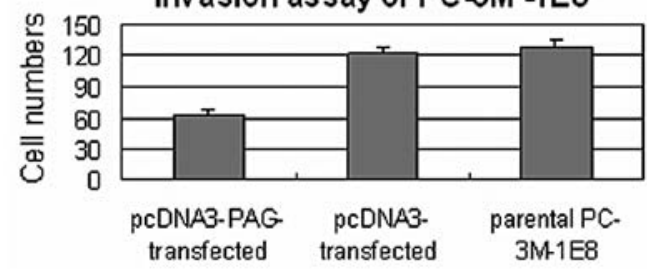

Invasion assay of DU145

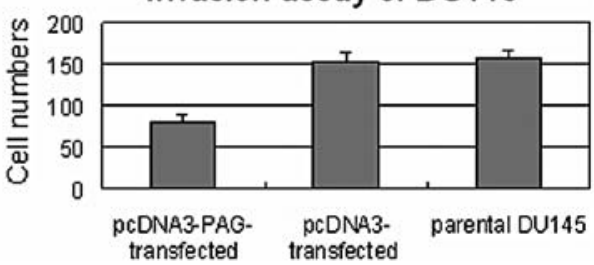

Figure 6. The results of invasion assay. The cell numbers passing through the matrigel and millipore membrane decreased in PAG-transfected PC-3M-1E8 and DU145 cells.

A

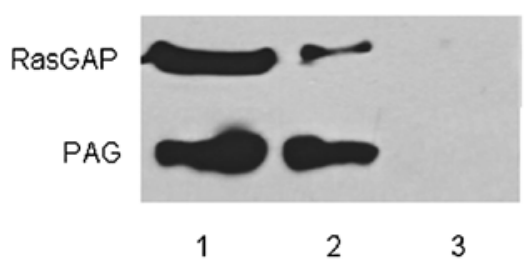

B The expression of GST-Raf1RBD in GST pull-down

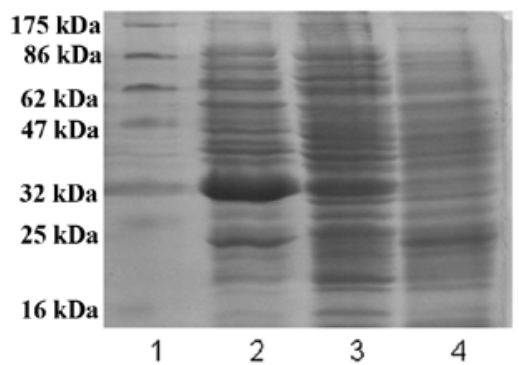

C

\section{Expression of RAS-GTP}
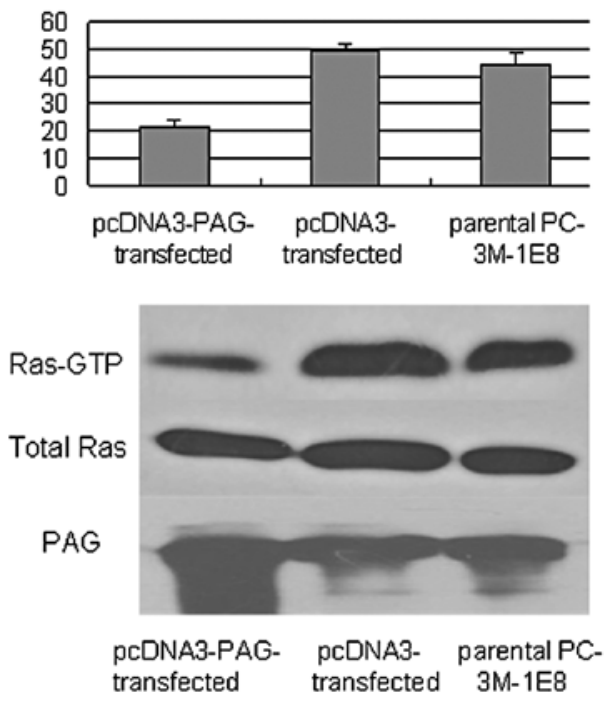

Figure 7. (A) The results of immunoprecipitation. Lane 1, the total protein of PC-3M-1E8. Lane 2, the immunoprecipitate by anti-PAG immunosorbent of PC-3M-1E8 after transfection. Lane 3, mouse IgG replaced the anti-PAG immunosorbent as the control. RasGAP appeared in the position of $91 \mathrm{kDa}$ showed by western blot in lanes 1 and 2. (B) The results of GST-pull down. Lane 1, standard protein marker. Lane 2, the expression of pGEX-GST-Raf1-RBD with the induction of IPTG. Lane 3, the expression of pGEX-GST-Raf1-RBD without induction of IPTG. Lane 4, the protein expression of pGEX with induction of IPTG. (C) The expression of Ras-GTP and total Ras in PC-3M-1E8 cell groups. The expression level of Ras-GTP decreased after exogenous expression of PAG in PC-3M-1E8 cells.

that exogenously expressed PAG. The results showed that the number of PC-3M-1E8 and DU145 cells passed the matrigel and millipore membrane was reduced after exogenous expression of PAG $(\mathrm{P}<0.5$, Fig. 6), proving that PAG inhibits the invasion of prostate cancer cells.
PAG interacts with RasGAP and inhibits the activation of Ras signaling in prostate cancer cells. To elucidate the potential mechanism by which PAG modulates the proliferation and invasion of prostate cancer cells, we decided to characterize the interacting partners of PAG in prostate cancer cells. Since 


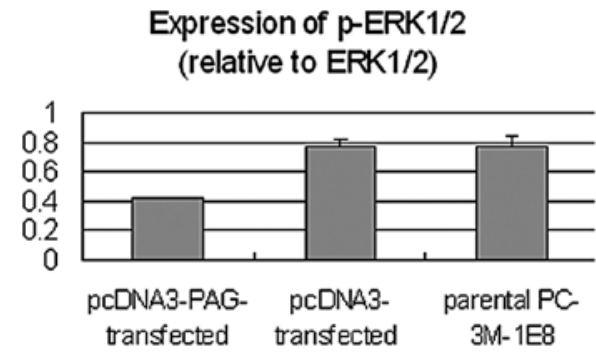

\section{Expression of cyclin D1}

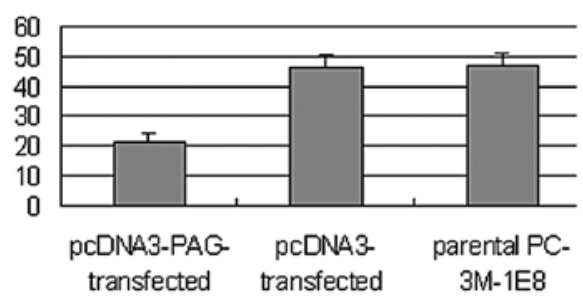

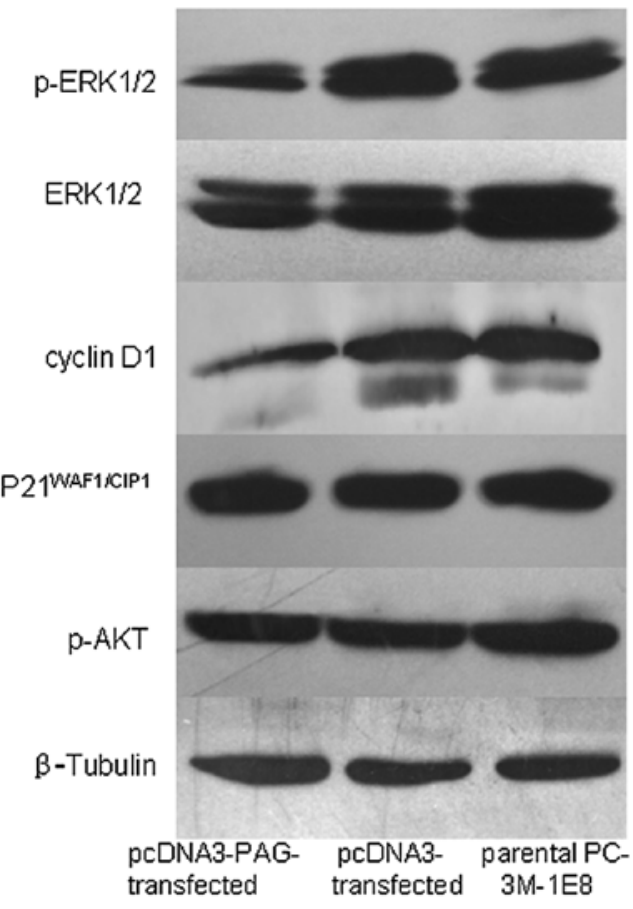

Figure 8. The expression of downstream proteins of Ras pathway including p-ERK1/2, ERK1/2, cyclin D1, P21 ${ }^{\text {WAFI/CIP1 }}$ and p-AKT detected by western blotting in PC-3M-1E8 cell groups. After exogenous expression of PAG in PC-3M-1E8 cell, the expression of p-ERK1/2 and cyclin D1 reduced.

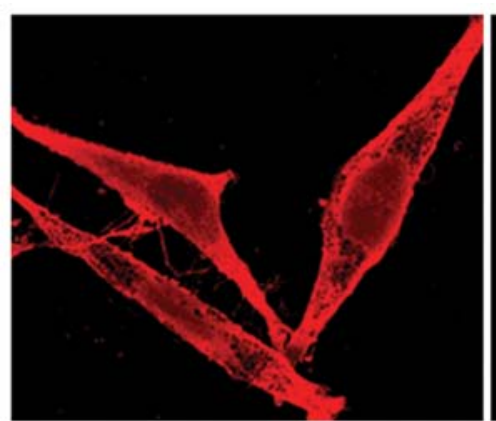

pcDNA3-PAG-transfected

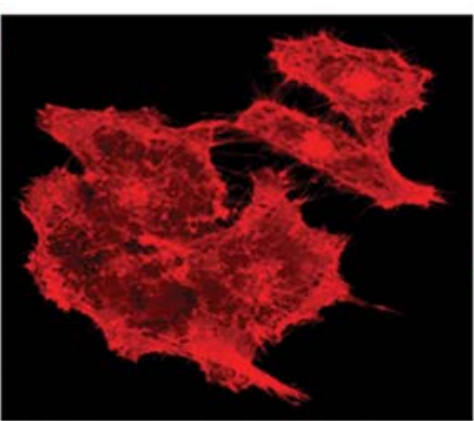

pcDNA3-transfected

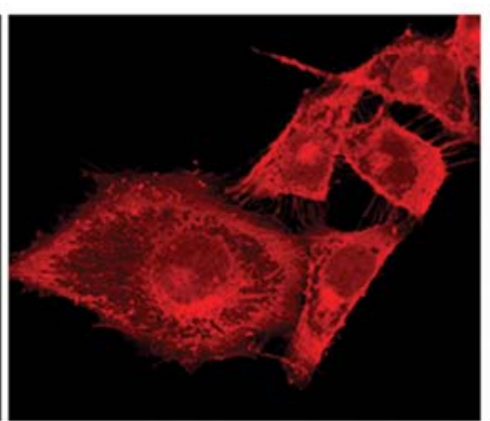

parental PC-3M-1E8

Figure 9. Phalloidine labeled by TRITC stained the intracellular F-actin and outlined the cell morphology of PC-3M-1E8 cell groups. Exogenous expression of PAG in PC-3M-1E8 cells led to reduced formation of pseudopodia and disrupted structure of intracellular F-actin.

RasGAP was suggested to bind directly to PAG in T lymphocyte, immunoprecipitation experiment was performed to examine the interaction between PAG and RasGAP in PC-3M-1E8 cells. The results showed that endogenous RasGAP could be co-precipitated with PAG in PC-3M-1E8 cells that stably transfected with PAG expression plasmid by PAG antibody but not mouse IgG control (Fig. 7A). Having established that PAG interacted with RasGAP, next we examined whether PAG could modulate the activation of Ras. To this end, we performed GST pull-down assay in which the pGEX-Raf1-Ras-binding domain (RBD) encoding amino acids 1-140 of c-Raf-1 fused to GST could specially bind the activated Ras (Ras-GTP). After the induction of IPTG, the GST-Raf1-RBD fusion protein with the molecular of $33 \mathrm{kDa}$ was highly expressed in JM109 (Fig. 7B). GST pull-down assay demonstrated that the activated Ras (Ras-GTP) was reduced in PC-3M-1E8 cells that stably transfected with PAG expression plasmid $(\mathrm{P}<0.5)$, but the amount of total Ras did not change, compared to the controls (Fig. 7C).

To provide further evidence that PAG modulates Ras signal pathway, we examined the levels of components of Ras signaling by western blot and found that the levels of p-ERK1/2 and cyclin D1, two crucial factors involved in cell proliferation, were decreased notably accompanied with the increased expression of PAG in PC-3M-1E8 cells $(\mathrm{P}<0.5)$. However, the levels of P21 $1^{\mathrm{WAF} 1 / \mathrm{CIP} 1}$ and $\mathrm{p}-\mathrm{AKT}$ did not show significant changes (Fig. 8). Collectively, these data strongly suggest that PAG inhibits the activation of Ras signaling in prostate cancer cells by interacting with RasGAP.

PAG modulates intracellular F-actin in prostate cancer cells. Cell skeleton is crucially involved in cell motility and invasion. 
Therefore, we examined the effects of PAG on the skeleton remodeling and morphology of PC-3M-1E8 cells. Our results showed that exogenous expression of PAG in PC-3M-1E8 cells led to reduced formation of pseudopodia and disrupted structure of intracellular F-actin as detected by TRITC staining under a confocal microscope (Fig. 9).

\section{Discussion}

In this study we focused on PAG, a candidate gene that was selected from cDNA microarray screening of genes up-regulated or down-regulated in high metastatic subline PC-3M-1E8 cells. PAG is a transmembrane adaptor protein without any enzymatic activity but acts as a scaffold in the signal transduction. Interestingly, the 397-amino acid cytoplasmic domain of PAG contains ten tyrosine residues which are the substrates of $\mathrm{Src}$ family kinases (13). When the tyrosine residues of PAG are phosphorylated, it could recruit proteins with special domains such as $\mathrm{SH} 2$ domain to the membrane and subsequently induce their phosphorylation or dephosphorylation to regulate the signal transduction in T lymphocyte (14). Although PAG has been shown as an important negative regulator of immunoreceptor signaling in T lymphocyte, the role of PAG in tumor cells remains largely unexplored.

First, we confirmed that the expression of PAG at mRNA and protein levels in the high metastatic PC-3M-1E8 and DU145 cells was significantly lower than that in the low metastatic PC-3M-2B4 cells, in agreement with the results of cDNA microarrays we previously reported. Next we restored the expression of PAG in PC-3M-1E8 and DU145 cells by establishing stable transfection of pcDNA3-PAG expression vector and examined the resulting biological effects. Our results demonstrated that reconstitution of PAG in high metastatic PC-3M-1E8 and DU145 cells led to reduced proliferation through cell cycle arrest, decreased anchorage-independent growth and reduced invasion ability of PC-3M-1E8 and DU145 cells in vitro. Collectively, these data provide important evidence that PAG may play an important role in inhibiting the proliferation and invasion of prostate cancer cells.

To further explore the potential mechanism underlying PAG-induced inhibition of the proliferation and invasion of prostate cancer cells, we characterized the interaction of PAG with RasGAP, a known binding partner of PAG in $\mathrm{T}$ lymphocytes. Immuno-coprecipitation assay proved that PAG interacted with RasGAP in prostate cancer cells, indicating the potential link of PAG with Ras signaling. To address this possibility we performed GST pull-down utilizing Raf1-RBD domain to detect the level of activated Ras and found that Ras activation was down-regulated after the reconstitution of PAG in PC-3M-1E8 cells although the total amount of Ras showed no significant change. In addition, western blot analysis demonstrated that the levels of downstream proteins of Ras signaling such as p-ERK1/2 and cyclin D1 were decreased upon the reconstitution of PAG in PC-3M-1E8 cells. Taken together, we speculate that PAG interacts with RasGAP in prostate cancer cells to recruit RasGAP to the cell membrane where it hydrolyzes GTP to GDP and reduces the level of activated Ras, ultimately suppressing the activation of ERK1/2, cyclin D1 and other effectors of Ras signal pathway.
In addition, we found that the formation of pseudopodia in the cellular surface and the structure of F-actin were disturbed after the reconstitution of PAG in PC-3M-1E8 cells. Previous studies showed that PAG could inhibit the movement of the membrane lipid raft and reduce the formation of pseudopodia in T lymphocyte $(15,16)$. Brdickova et al reported that PAG could interact with EBP50 to modulate F-actin (3). The formation of pseudopodia has been proposed as the early and critical step for the migration of tumor cells into and out of the vascular space and metastasis to the distant organs (17). Therefore, we assume that the redistribution of F-actin and impairment of pseudopodia of cellular surface could directly lead to the decreased invasion and metastasis potential of PC-3M-1E8 cells.

In conclusion, our data demonstrated the increased expression of PAG could inhibit the proliferation and invasion potential of prostate cancer cells in vitro by suppressing the activations of Ras and downstream effectors such as ERK and cyclin D1 through the interaction with RasGAP. Morphologically, we observed that the increased expression of PAG could diminish the formation of pseudopodia on cellular surface and lead to the redistribution of the intracellular F-actin in PC-3M-1E8 cells. Taken together, these results suggested that PAG acts to inhibit the development and metastasis of prostate cancer cells and represents a novel therapeutic target for prostate cancer.

\section{Acknowledgements}

This study was supported by Nation 973 basal research development assistance programs (2009CB521805) of China.

\section{References}

1. Liu YX, Zheng J and Fang WG: Isolation and characterization of human prostate cancer cell subclones with different metastatic potential. Chin J Pathol 28: 361-364, 1999.

2. Svec A: Phosphoprotein associated with glycosphingolipidenriched microdomains/Csk-binding protein: a protein that matters. Pathol Res Pract 204: 785-792, 2008.

3. Brdickova N, Brdicka T, Andera L, Spicka J, Angelisova P, Milgram SL and Horejsi V: Interaction between two adapter proteins, PAG and EBP50: a possible link between membrane rafts and actin cytoskeleton. FEBS Lett 507: 133-136, 2001.

4. Kawabuchi M, Satomi Y, Takao T, Shimonishi Y, Nada S, Nagai K, Tarakhovsky A and Okada M: Transmembrane phosphoprotein Cbp regulates the activities of Src-family tyrosine kinases. Nature 404: 999-1003, 2000.

5. Takeuchi S, Takayama Y, Ogawa A, Tamura K and Okada M: Transmembrane phosphoprotein cbp positively regulates the activity of the carboxyl-terminal src kinase Csk. J Biol Chem 275: 29183-29186, 2000.

6. Brdicka T, Pavlistova D, Leo A, Bruyns E, Korinek V, Angelisova P, Scherer J, Shevchenko A, Hilgert I, Cerny J, Drbal K, Kuramitsu Y, Kornacker B, Horejsi V and Schraven B: Phosphoprotein associated with glycosphingolipid-enriched microdomains (PAG), a novel ubiquitously expressed transmembrane adaptor protein, binds the protein tyrosine kinase csk and is involved in regulation of $\mathrm{T}$ cell activation. J Exp Med 191: 1591-1604, 2000.

7. Torgersen KM, Vang T, Abrahamsen H, Yaqub S, Horejsi V, Schraven B, Rolstad B, Mustelin T and Tasken K: Release from tonic inhibition of $\mathrm{T}$ cell activation through transient displacement of C-terminal Src kinase (Csk) from lipid rafts. J Biol Chem 276: 29313-29318, 2001.

8. Jiang LQ, Feng X, Zhou W, Knyazev PG, Ullrich A and Chen Z: Csk-binding protein (Cbp) negatively regulates epidermal growth factor-induced cell transformation by controlling Src activation. Oncogene 25: 5495-5506, 2006.

9. Smida M, Posevitz-Fejfar A, Horejsi V, Schraven B and Lindquist JA: A novel negative regulatory function of the phosphoprotein associated with glycosphingolipid-enriched microdomains: blocking Ras activation. Blood 110: 596-615, 2007. 
10. Durrheim GA, Garnett D, Dennehy KM and Beyers AD: Thy-1 associated pp85-90 is a potential docking site for SH2 domaincontaining signal transduction molecules. Cell Biol Int 25: 33-42, 2001.

11. Sanchez-Margalet V and Najib S: Sam68 is a docking protein linking GAP and PI3K in insulin receptor signaling. Mol Cell Endocrinol 183: 113-121, 2001.

12. Huang KT, Chen YH and Walker AM: Inaccuracies in MTS assays: major distorting effects of medium, serum albumin, and fatty acids. Biotechniques 37: 406-412, 2004.

13. Simeoni L, Smida M, Posevitz V, Schraven B and Lindquist JA Right time, right place: the organization of membrane proximal signaling. Semin Immunol 17: 35-49, 2005.

14. Cloutier JF, Chow LM and Veillette A: Requirement of the SH3 and $\mathrm{SH} 2$ domains for the inhibitory function of tyrosine protein kinase p50csk in T lymphocytes. Mol Cell Biol 15: 5937-5944, 1995.
15. Itoh K, Sakakibara M, Yamasaki S, Takeuchi A, Arase H, Miyazaki M, Nakajima N, Okada M and Saito T: Cutting edge: negative regulation of immune synapse formation by anchoring lipid raft to cytoskeleton through Cbp-EBP50-ERM assembly. J Immunol 168: 541-544, 2002.

16. Harder T and Simons K: Clusters of glycolipid and glycosylphosph atidylinositol-anchored proteins in lymphoid cells: accumulation of actin regulated by local tyrosine phosphorylation. Eur J Immunol 29: 556-562, 1999.

17. Condeelis JS, Wyckoff JB, Bailly M, Pestell R, Lawrence D, Backer J and Segall JE: Lamellipodia in invasion. Semin Cancer Biol 11: 119-128, 2001. 